\title{
Estudio de la prevalencia de la infección por Trypanosoma cruzi en zarigüeyas (Didelphis albiventris) en Santiago del Estero, Argentina ${ }^{1}$
}

\author{
Nicolás J. Schweigmann, ${ }^{2}$ Silvia Pietrokovsky, ${ }^{2}$ Victoria Bottazzi, ${ }^{2}$ \\ Osvaldo Conti, ${ }^{2}$ Marcos A. Bujas ${ }^{3}$ y Cristina Wisnivesky-Colli ${ }^{2}$
}

RESUMEN Entre los principales reservorios silvestres de Trypanosoma cruzi se encuentran las zarigüeyas del género Didelphis, ampliamente distribuidas por el continente americano. En Amamá y Trinidad, Provincia de Santiago del Estero, Argentina, Didelphis albiventris es el marsupial más frecuente. Su población se renueva cada año y normalmente hay dos períodos reproductivos: uno a principios de la primavera y otro a principios del verano. Estas dos camadas son destetadas y abandonan la bolsa marsupial para incorporarse a la población, la primera (G1) a principios del verano y la segunda (G2) a principios del otoño. Entre 1988 y 1991 se estudiaron 409 individuos distintos de D. albiventris y los xenodiagnósticos revelaron que 35\% de ellos estaban infectados por T. cruzi. Se observaron ciclos de renovación anual de la infección con prevalencias que oscilaron entre 22 y 43\%. La adquisición del parásito ocurría a lo largo de todo el año, desde el verano hasta la primavera. La prevalencia de la infección aumentó con la edad. Los individuos G1 tuvieron tendencia a presentar mayores prevalencias que los G2, probablemente debido a un mayor tiempo de exposición a la transmisión. En las dos primeras categorías de edad, los individuos G2 mostraron mayores prevalencias que los G1, lo cual indica un aumento significativo de la intensidad de la transmisión durante el otoño. Las zarigüeyas deberían considerarse como una fuente potencial de ingreso de T. cruzi al ciclo doméstico.

La enfermedad de Chagas o tripanosomiasis americana, que afecta a apro-

\footnotetext{
1 Esta investigación recibió apoyo económico del Consejo Nacional de Investigaciones Científicas y Tecnológicas (CONICET) y de la Universidad de Buenos Aires.

2 Universidad de Buenos Aires, Facultad de Ciencias Exactas y Naturales, Departamento de Ciencias Biológicas, Unidad de Ecología de Reservorios y Vectores de Parásitos. Enviar toda la correspondencia a Nicolás J. Schweigmann a la siguiente dirección postal: Ciudad Universitaria, Pabellón 2, $4^{\circ}$ piso, Departamento de Ciencias Biológicas, 1428 Buenos Aires, Argentina. Correo electrónico: nicolas@bg.fcen.uba.ar

3 Servicio de Hemoterapia, Hospital Italiano, Buenos Aires, Argentina.
}

ximadamente 18 millones de personas en América Latina, es una parasitosis causada por el flagelado Trypanosoma cruzi. En las zonas endémicas, T. cruzi se transmite especialmente por la vía vectorial a través de las deyecciones de triatominos (Hemiptera: Reduviidae: Triatominae) (1).

Aunque originalmente la tripanosomiasis americana fue una enzootia, en la actualidad no solo existen ciclos silvestres de transmisión, sino también ciclos peridomésticos y domésticos con características propias según cada lugar. En el ambiente silvestre y peri- doméstico, las zarigüeyas del género Didelphis (D. virginiana, D. marsupialis y D. albiventris) presentan importantes prevalencias de infección por T. cruzi y son consideradas como los hospedadores de mantenimiento del parásito $(1,2)$. La distribución geográfica de este género es muy amplia, desde el nordeste de Canadá (3) hasta el norte de la Patagonia (4), y se han encontrado ejemplares infectados por $T$. cruzi en todos los países estudiados (2). Su susceptibilidad a la infección es muy alta (5), tienen parasitemia persistente y presentan gran capacidad para 
infectar vectores triatominos (6). Aparentemente, la infección no les provoca enfermedad y algunos autores sugieren que esto evidenciaría una asociación muy antigua entre los marsupiales y el parásito (7). Las zarigüeyas son animales omnívoros, de hábitos nocturnos y merodeadores, que suelen acercarse a las viviendas en busca de alimento $(3,4)$. Pueden así adquirir la infección al ingerir triatominos o transmitirla al servir como fuente de sangre para estos, constituyéndose en un nexo entre los ciclos silvestre y doméstico $(1,6,8)$.

D. albiventris es exclusivamente sudamericano y su mapa de distribución tiene forma de " $Y$ " (figura 1), cuyo brazo occidental corresponde a las alturas medias de los Andes (Bolivia, Colombia, Ecuador y Perú); la rama oriental se extiende por la costa de Brasil (Ceará, Pernambuco) y el eje inferior corresponde a Argentina, Paraguay y Uruguay, hasta aproximadamente los $40^{\circ}$ de latitud sur en el valle

FIGURA 1. Distribución geográfica (zona sombreada) de la zarigüeya Didelphis albiventris y localidades del área de estudio

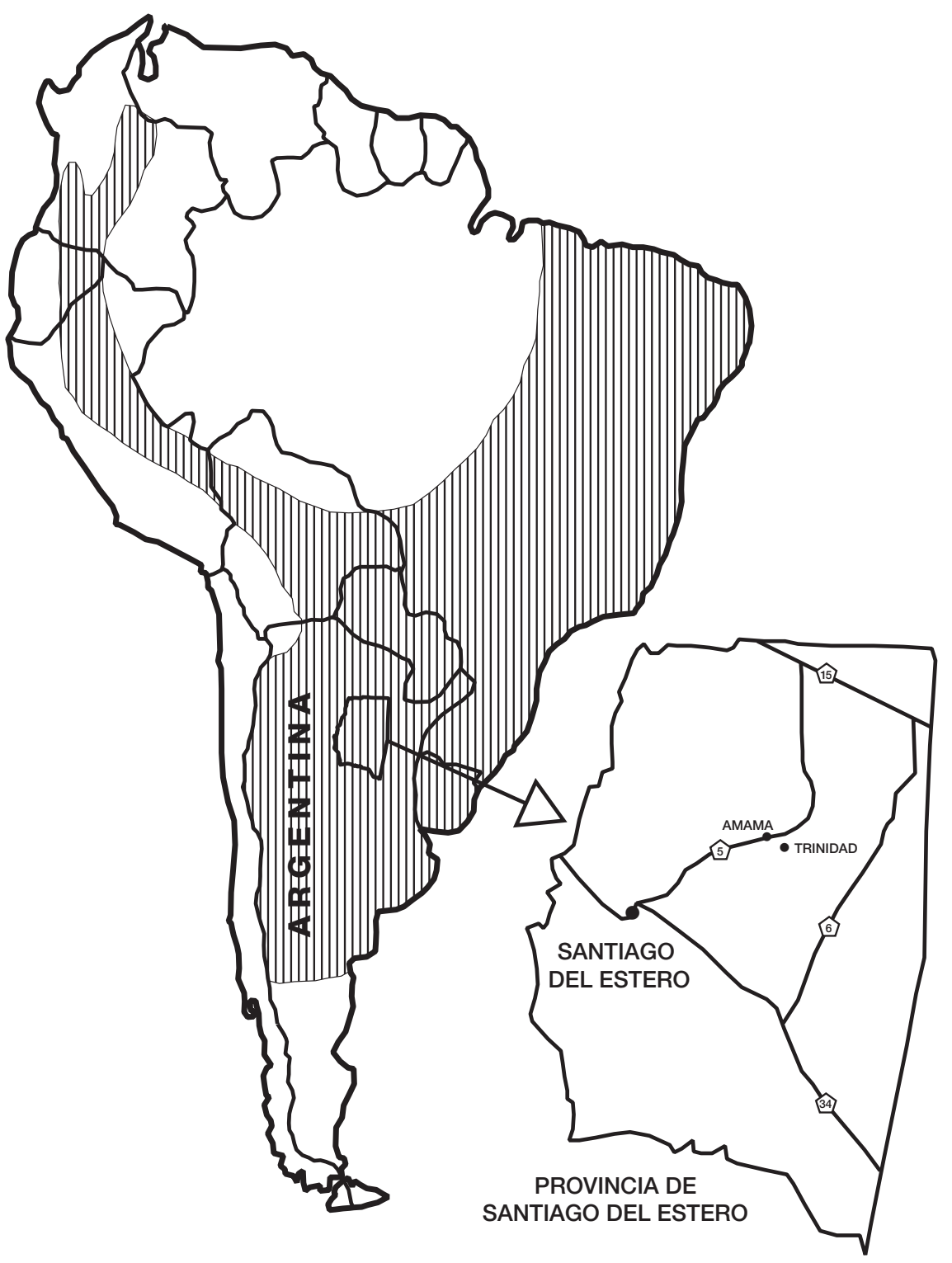

del río Negro en la Patagonia. Al igual que $D$. virginiana, $D$. albiventris vive en zonas templadas de bosques, praderas o pastizales $(2,4)$.

En Argentina, D. albiventris (localmente llamada comadreja overa) es la especie de zarigüeya más abundante y, además, es frecuente en los límites entre las zonas rurales y la periferia de las ciudades. En la Provincia de Santiago del Estero, donde la enfermedad de Chagas es altamente endémica, Schweigmann (2) estudió la dinámica poblacional de $D$. albiventris y comprobó que este marsupial tiene una longevidad no superior a 1 a 1,5 años y que su mortalidad es independiente de la edad. Existen dos períodos reproductivos: el primero entre principios de septiembre y mediados de octubre, coincidiendo con la época de mayor fotoperíodo y temperatura, y el segundo entre fines de noviembre y fines de diciembre. En consecuencia, se producen dos períodos de destete: a principios del verano (individuos del grupo 1: G1) y a fines del verano y principios del otoño (individuos del grupo 2: G2). Los individuos de uno y otro grupo se desarrollan en distintas condiciones climáticas de fotoperíodo y abundancia de alimentos. Las categorías de edad se pueden discriminar fácilmente a través de la observación de la erupción y el desgaste dentario (2). Los individuos deben sobrevivir hasta la primavera siguiente para entrar en su primer estro.

Hay datos que indican la existencia de altos niveles de infección en poblaciones de $D$. albiventris de Santiago del Estero. Canal Feijóo (9) verificó que $49 \%$ de los 69 ejemplares capturados en los alrededores de la capital presentaban flagelados "tipo cruzi" en muestras de sangre periférica. Posteriormente, al estudiar la infección de mamíferos silvestres por $T$. cruzi en un bosque de la misma zona de muestreo del presente trabajo, Wisnivesky-Colli et al. (10) comprobaron que, entre las 19 especies analizadas por xenodiagnóstico, $D$. albiventris mostraba la mayor prevalencia de infección (32\%). A pesar de que las zarigüeyas son consideradas como los principales hospedadores silvestres de T. cruzi, el único es- 
tudio que ha relacionado la dinámica poblacional de estos marsupiales con la dinámica de transmisión del parásito ha sido el realizado por Telford y Tonn (11) sobre D. marsupialis en los llanos altos venezolanos.

El objetivo de este estudio longitudinal consistió en investigar la prevalencia de la infección por T. cruzi en poblaciones naturales de $D$. albiventris de una zona endémica de la enfermedad de Chagas en Argentina, tomando en consideración la edad de las zarigüeyas y las estaciones del año.

\section{MATERIALES Y MÉTODOS}

\section{Área de estudio}

Los muestreos se realizaron en el bosque que rodea a las localidades rurales de Amamá y Trinidad, distantes $9 \mathrm{~km}$ entre sí y situadas en el Departamento de Moreno, Provincia de Santiago del Estero $\left(27^{\circ} 12^{\prime} 30^{\prime \prime} \mathrm{S}, 6^{\circ} 02^{\prime}\right.$ $30^{\prime \prime} \mathrm{W}$ ), Argentina (figura 1). La zona está incluida en el Distrito Occidental Seco de la Región Chaqueña (12) y el paisaje se caracteriza por un bosque secundario formado por árboles altos de madera dura (Schinopsis lorentzii y Aspidosperma quebracho blanco), árboles de tamaño intermedio (Prosopis spp., Zizyphus mistol), arbustos, cactáceas y bromeliáceas. El clima es semiárido, con una estación seca en otoño e invierno. La temperatura media anual es de $22^{\circ} \mathrm{C}$; enero y febrero son los meses más cálidos y julio el más frío. La humedad relativa anual varía entre 55 y $68 \%$, y la precipitación anual media es de $740 \mathrm{~mm}$.

\section{Captura de las zarigüeyas}

Los ejemplares de $D$. albiventris se capturaron entre principios de 1988 y finales de 1991. Las capturas se efectuaron con trampas tipo National, cebadas con grasa de vaca mezclada con vísceras o con alimento equilibrado para ratones y esencia de banana. También se usaron trampas tipo cepo y capturas por extracción manual de los refugios.

\section{Examen de las zarigüeyas}

Los individuos capturados fueron llevados a un laboratorio de campo, donde se los anestesió con $2 \mathrm{mg} / \mathrm{kg}$ de una mezcla de acepromazina (Acedan ${ }^{\circledR}$, Holliday-Scott) y clorhidrato de ketamina (Ketalar ${ }^{\circledR}$, Parke-Davis) por vía intramuscular. Para determinar la edad, primero se intentó emplear la relación longitud/peso utilizada por Telford y Tonn (11), pero al no encontrarse correlación entre estas variables, finalmente se decidió usar la fórmula dentaria de los maxilares superiores, que considera la erupción y el desgaste dentario (2) (figura 2).

$\mathrm{El}$ análisis parasitológico se realizó solamente en animales de vida libre no lactantes, mediante la técnica de xenodiagnóstico (13), recomendada por Zeledón et al. (14) como la más sensible para detectar la infección por T. cruzi en D. marsupialis de Costa Rica. Sobre el vientre de cada animal se aplicaron durante 15 minutos dos cajas con cinco triatominos cada una. Los insectos, Triatoma infestans de tercero o cuarto estadio, provenían del insectario del Servicio Nacional de Chagas, Córdoba, y tras el xenodiagnóstico fueron remitidos a nuestro laboratorio en Buenos
Aires. Antes de ser liberados, los marsupiales fueron marcados para su identificación posterior.

\section{Examen parasitológico de los triatominos}

Las heces de los triatominos se observaron al microscopio (400x) 30, 60 y 90 días después de la ingesta sanguínea. Se consideró que una zarigüeya era positiva cuando al menos un triatomino estaba infectado. En un estudio previo (10) se determinó que los tripanosomas aislados eran $T$. cruzi por criterios biológicos y bioquímicos (15). La caracterización previa del aislado de T. cruzi que circulaba entre las zarigüeyas locales mostró en el zimograma electroforético un patrón clasificado como Z10, íntimamente relacionado con los zimodemas Z2 y Z12 aislados a partir de humanos en Argentina (9).

\section{Análisis estadísticos}

Las variaciones estacionales de la prevalencia de la infección por T. cruzi en las poblaciones de D. albiventris se analizaron con una prueba de propor-

FIGURA 2. Edad de la zarigüeya Didelphis albiventris según la erupción y el desgaste dentario

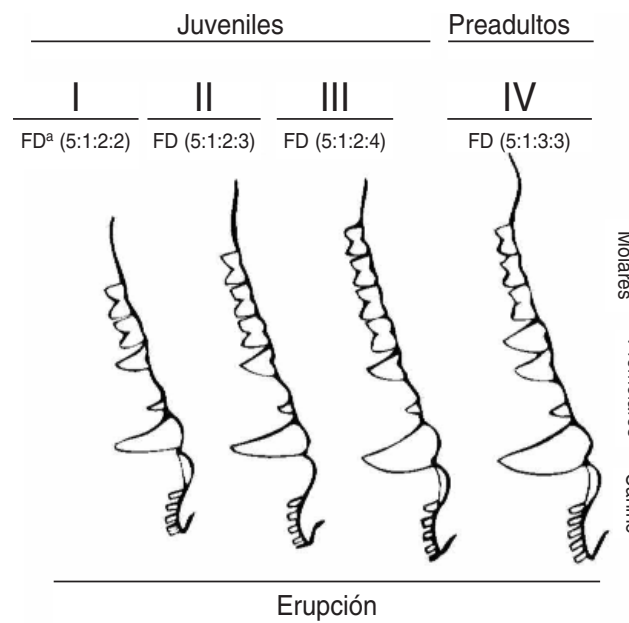

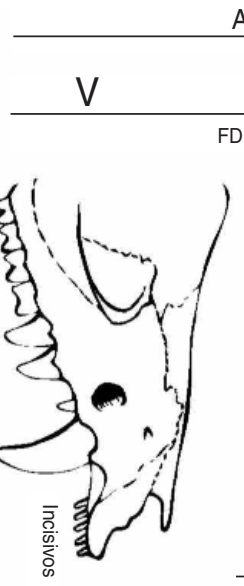

o

Edades: I — erupción de 2 premolares y dos molares, el primero decíduo; II: se agrega un tercer molar; III: se agrega un cuarto molar; IV: el primer molar (decíduo) es reemplazado por el tercer premolar; V: erupción del ultimo molar; VI y VII: cúspides de caninos, premolares y molares desgastadas.

a $F D$ fórmula dentaria 
CUADRO 1. Prevalencias estacionales de infección por Trypanosoma cruzi en la zarigüeya Didelphis albiventris. Santiago del Estero, Argentina, 1988-1991

\begin{tabular}{lccccc}
\hline \multirow{2}{*}{ Año del estudio } & \multicolumn{4}{c}{ Número de D. albiventris infectados/examinados (porcentaje) } \\
\cline { 2 - 6 } & Verano & Otoño & Invierno & Primavera & Total \\
\hline \multirow{2}{*}{1988} & $2 / 12$ & $10 / 48$ & $3 / 14$ & $5 / 15$ & $20 / 89$ \\
& $(17 \%)$ & $(21 \%)$ & $(21 \%)$ & $(33 \%)$ & $(22 \%)$ \\
1989 & - & $13 / 39$ & - & $3 / 6$ & $16 / 45$ \\
& & $(33 \%)$ & & $(50 \%)$ & $(36 \%)$ \\
1990 & $3 / 24$ & $22 / 58$ & $37 / 84$ & $25 / 37$ & $87 / 203$ \\
& $(13 \%)$ & $(38 \%)$ & $(44 \%)$ & $(68 \%)$ & $(43 \%)$ \\
1991 & $2 / 14$ & $13 / 41$ & - & $9 / 17$ & $24 / 72$ \\
& $(14 \%)$ & $(32 \%)$ & & $(53 \%)$ & $(33 \%)$ \\
Promedio & $7 / 50$ & $58 / 186$ & $40 / 98$ & $42 / 75$ & $147 / 409$ \\
estacional & $(14 \%)$ & $(31 \%)$ & $(41 \%)$ & $(56 \%)$ & $(36 \%)$ \\
\hline
\end{tabular}

ciones múltiples, y su incremento con la edad de los animales mediante un análisis de gradiente de proporciones para muestras ordenadas cuantitativamente (16)

\section{RESULTADOS}

Entre 1988 y 1991 se capturaron 409 individuos, de los cuales 147 (36\%) estaban infectados. Las prevalencias anuales oscilaron entre 22 y $43 \%$ (cuadro 1). El análisis de las prevalencias registradas en las diferentes estaciones del año solo mostró dos incrementos significativos $(P<0,005)$ : uno entre el verano y el otoño, y el otro entre el invierno y la primavera (cuadro 1). Durante el verano las capturas estaban formadas por individuos jóvenes recién destetados y por una pequeña proporción de hembras portadoras de lactantes; en esta estación se registraron las menores prevalencias en los años estudiados. Durante el otoño, la abundancia poblacional fue máxima, debido a la superposición de los juveniles de los dos grupos de destete y a la presencia de algunas hembras adultas, y la prevalencia media aumentó en $17 \%$. En invierno ya no se detectaron adultos del año anterior y solo estaban presentes los individuos destetados en el mismo año; aunque la prevalencia media aumentó en $10 \%$ en comparación con el otoño, no se detectaron diferencias entre el otoño y el invierno al comparar cada uno de los años analizados: 1988 y 1990. La máxima prevalencia media $(56 \%)$ se detectó en primavera, cuando los individuos de ambos grupos ya habían alcanzado las edades IV y V (preadultos y adultos respectivamente) y entraban en su primer período reproductivo.

Las prevalencias de infección analizadas por edades en cada año se muesde 1991). tran en la figura 3. Además se incluyen las prevalencias totales para cada edad, calculadas como el total de individuos infectados dividido por el total de individuos examinados en el conjunto de los cuatro años de muestreo. El ajuste de estas prevalencias en función de la edad no difiere de un modelo lineal $(P<0,05)$. Por otra parte, las prevalencias aumentaron de forma significativa con la edad $(P<0,05)$, desde un promedio de $5,7 \%$ en los individuos más jóvenes (edad I) hasta $70,8 \%$ en los más viejos (edades VIVII). La edad III fue la que mostró prevalencias más heterogéneas en los distintos años analizados.

$\mathrm{Al}$ separar los resultados por grupo de destete, las prevalencias estacionales de los individuos G1, destetados en verano, superaron en todos los casos a las de los individuos G2, destetados en otoño (figura 4). Sin embargo, cuando se analizaron las prevalencias de cada grupo en función de la edad (figura 5), los individuos de edad I y II del grupo G2 presentaron mayores prevalencias que los de la misma edad del grupo G1.

FIGURA 3. Prevalencia de infección por Trypanosoma cruzi en las distintas categorías de edad de la zarigüeya Didelphis albiventris. Santiago del Estero, Argentina, 1988-1991a

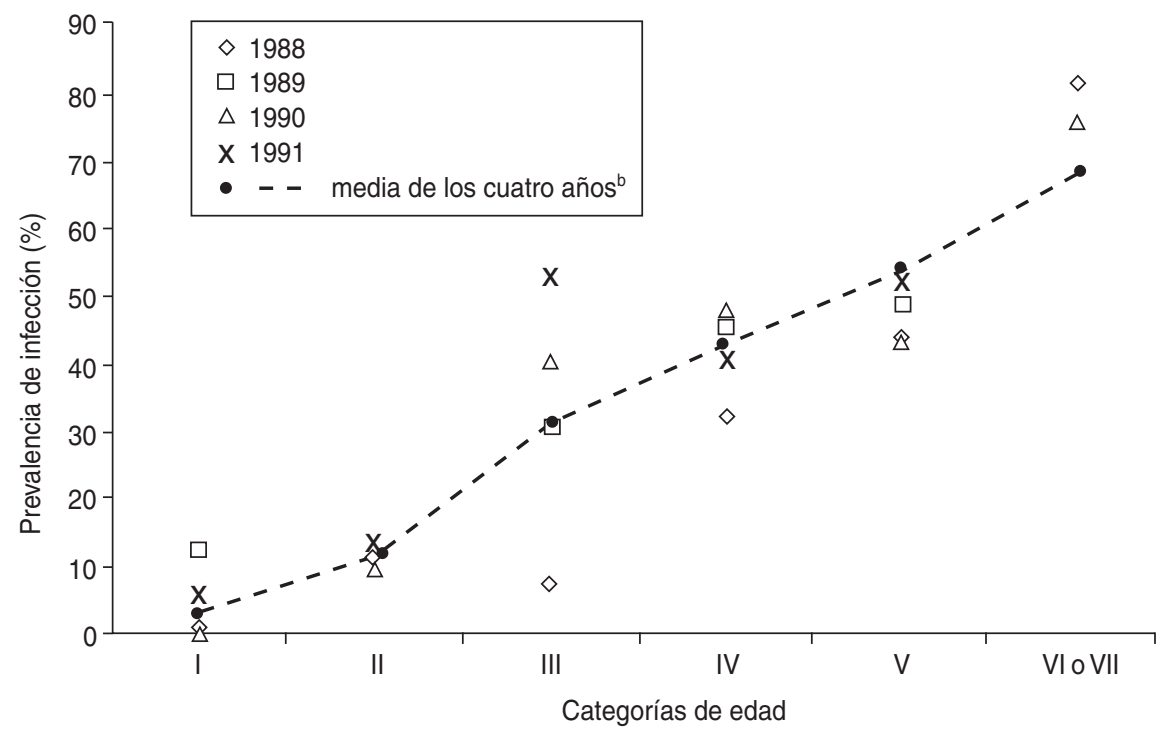

a Se omiten las prevalencias de las muestras de tamaño inferior a 6 individuos (categorías II y VI-VII del año 1989 y VI-VII

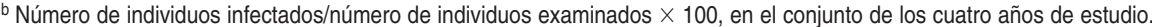


FIGURA 4. Comparación de las prevalencias de infección por Trypanosoma cruzi entre los grupos de destete G1 y G2 de la zarigüeya Didelphis albiventris según las estaciones del año. Santiago del Estero, Argentina, 1988-1991

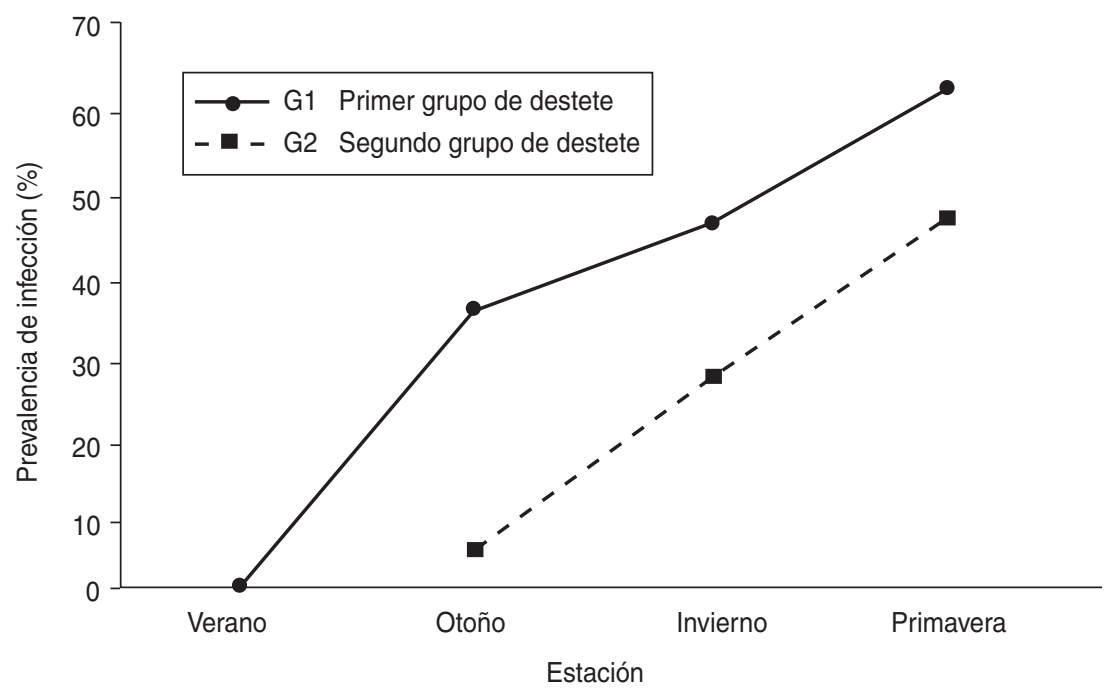

\section{DISCUSIÓN}

En una revisión bibliográfica (2) que incluyó 41 estudios sobre la prevalencia de la infección por T. cruzi en las tres especies del género Didelphis del continente americano, se registró una

FIGURA 5. Comparación de las prevalencias de infección por Trypanosoma cruzi entre los grupos de destete G1 y G2 de la zarigüeya Didelphis albiventris según la edad. Santiago del Estero, Argentina, 1988-1991

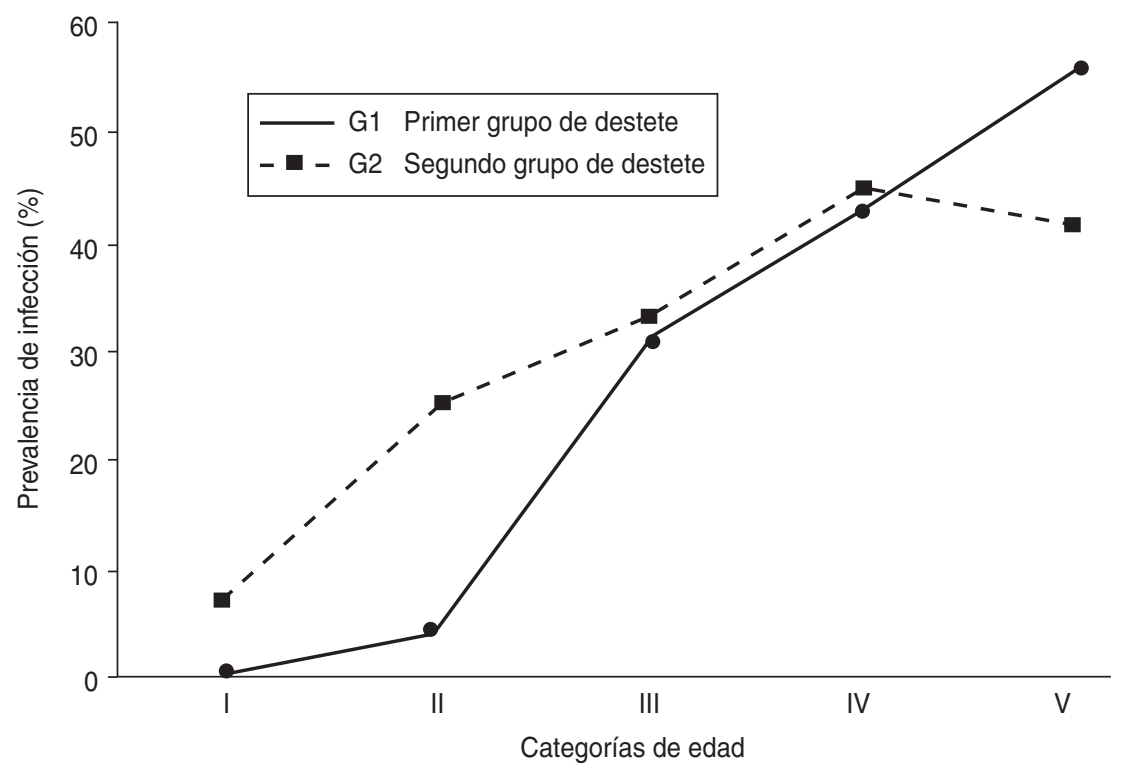

tico, D. marsupialis presentó la cifra más elevada $(42,6 \%$; 561/1317) y D. albiventris una prevalencia intermedia $(28,7 \%$; 133/464). Las prevalencias medias registradas en los ejemplares de D. albiventris de Santiago del Estero examinados en el presente estudio están comprendidas dentro de estos límites.

El hecho de que la longevidad de D. albiventris no supere los $12-18$ meses, junto con el hallazgo de prevalencias anuales que oscilaron entre 22 y $43 \%$, sugieren que la dinámica de transmisión de T. cruzi en las poblaciones naturales de este marsupial en Santiago del Estero se renueva anualmente. Esto se diferencia de lo que ocurre en el ciclo doméstico, donde los perros, hospedadores de mantenimiento del parásito y más longevos que las zarigüeyas, mostraron parasitemias elevadas y persistentes (17) que aseguran la posibilidad de transmisión por varios años.

Por otra parte, el incremento de la prevalencia con la edad y en el transcurso de las estaciones sugiere la incorporación progresiva de nuevos individuos infectados por T. cruzi a la población de $D$. albiventris durante todo el año, excepto el invierno.

Los individuos G1 mostraron mayores prevalencias estacionales que los G2, debido posiblemente a un mayor tiempo de exposición a la transmisión. Sin embargo, cuando analizamos las prevalencias de las dos primeras categorías de edad de ambos grupos observamos que las de G2 son mayores que las de G1, lo cual indica que la intensidad de la transmisión aumenta en el otoño, estación durante la cual transcurren las edades juveniles de G2.

$\mathrm{El}$ incremento de la prevalencia durante las temporadas templadas a cálidas apoyaría la hipótesis de una transmisión vectorial, dado que en esa época aumenta la actividad de los triatominos (18). Por otra parte, el género Didelphis tiende a consumir una mayor proporción de insectos en edades tempranas (19). Esto explicaría parcialmente el hecho de que los individuos de las categorías de edad I y II alcancen mayores tasas de infección en otoño, cuando comienzan a escasear otros alimentos. 
Varios autores $(5,20,21)$ han presentado datos experimentales que demuestran que $D$. albiventris se podría infectar tanto a través de la vía vectorial clásica como por la ingesta de triatominos. Entre los biotopos que frecuenta $D$. albiventris también está presente Triatoma guasayana. Esta especie presenta bajos porcentajes de infección, pero las colonias tienen una distribución agregada, que aumentaría la probabilidad de transmisión (22). Se ha demostrado experimentalmente que la transmisión del parásito a este marsupìal es muy eficiente (5); por lo tanto, pocos contactos infectivos entre Triatoma guasayana y D. albiventris podrían originar las prevalencias observadas en campo.

Otra posibilidad es que D. albiventris se infecte a partir de Triatoma infestans, el único vector domiciliado en la Argentina, como lo sugieren los patrones isoenzimáticos de los tripanosomas aislados en zarigüeyas (10). Si bien Triatoma infestans está ausente en los biotopos silvestres, las zarigüeyas merodean en el ambiente domésticoperidoméstico y podrían entrar en contacto con este vector.
Con respecto a otros modos de transmisión, las bajas prevalencias registradas en edades tempranas restarían importancia a una posible transmisión vertical $(11,23,24)$. Este hecho se explicaría por la protección frente a la infección que las madres confieren a su descendencia a través de la inmunoglobulina G presente en la leche (25).

Por otro lado, se ha sugerido (26) que el contenido de las glándulas perianales sería una posible vía de transmisión directa entre zarigüeyas, tras el sorprendente hallazgo de epimastigotas y tripomastigotas metacíclicos en el lumen de dichas glándulas. Sin embargo, esta posibilidad no explicaría las altas prevalencias en zarigüeyas asociadas a triatominos silvestres con bajos porcentajes de infección. Un estudio realizado en la misma zona, con 56 ejemplares infectados de distintas edades y en distintas estaciones del año, demostró la ausencia de T. cruzi en las glándulas perianales (27). Otras vías de transmisión directa, como la orina o las heces (1), solo podrían justificar los altos porcentajes de infección si fueran muy eficientes, ya que estos marsupiales son solitarios y el único momento en que entran en estrecho contacto es durante el apareamiento en primavera.

Es de destacar la importancia sanitaria de $D$. albiventris como posible fuente de tripanosomas para el ser humano y sus animales domésticos. Durante el otoño y principios del invierno, coincidiendo con altas tasas de prevalencia de infección, los pobladores cazan zarigüeyas para comerciar su piel o alimentarse. Al faenarlas, pueden entrar en contacto con la sangre contaminada y es frecuente que las vísceras sirvan de alimento para los perros. En la eventual eliminación de los triatominos domiciliados, los programas gubernamentales deberían contemplar a las zarigüeyas y a los triatominos silvestres como un peligro potencial para la reintroducción del parásito.

Agradecimiento. Deseamos expresar nuestra gratitud a la familia Moyano, de Amamá, y a la familia Sosa, Don Ibera y Doña Leo, de Trinidad, por su valiosa ayuda durante nuestro trabajo de campo, y también a Mario Capovía por su inapreciable colaboración en la parte gráfica.

\section{REFERENCIAS}

1. World Health Organization. Control of Chagas disease. Geneva; 1991. (WHO/TRS/811).

2. Schweigmann NJ. Aspectos ecológicos de una población santiagueña de la comadreja overa (Didelphis albiventris) en relación con la transmisión del Trypanosoma cruzi [tesis doctoral]. Buenos Aires: Facultad de Ciencias Exactas y Naturales de la Universidad de Buenos Aires; 1994

3. Hunsaker D. Ecology of New World marsupials. En: Hunsaker D, ed. The biology of marsupials. 2a ed. New York: Academic Press; 1977. pp. 95-156.

4. Contreras JR. La comadreja overa. Buenos Aires: Centro Editor de América Latina; 1983. (Fauna Argentina, Publicación 11).

5. Schweigmann NJ, Pietrokovsky S, Bottazzi V, Conti O, Wisnivesky-Colli C. Interaction between Didelphis albiventris and Triatoma infestans in relation to Trypanosoma cruzi transmission. Mem Inst Oswaldo Cruz 1995;90(6): 679-682.

6. Schweigmann NJ, Pietrokovsky S, Conti O, Escosteguy M, Bottazzi V, Solarz N, et al. Infection of Triatoma guasayana, Triatoma sordida and Triatoma infestans by Trypanosoma cruzi from a naturally infected opossum. Mem Inst Oswaldo Cruz 1997;92(2):151-152.

7. Jansen AM. Aspects of successful host-parasite association: opossum and Trypanosoma cruzi. Mem Inst Oswaldo Cruz 1988;83(supl 1):491-496.

8. Barreto MP, Ribeiro RD. Reservatorios silvestres do Trypanosoma cruzi. Rev Inst Adolfo Lutz 1979;39:25-26.

9. Canal Feijóo EJ. Vectores, depósitos parasitarios y casos clínicos de la Enfermedad de Chagas en la Provincia de Santiago del Estero. En: Actas y Trabajos VI Congreso Nacional de Medicina, Córdoba, Argentina, 16 al 21 de octubre. 1939;3:103-113.

10. Wisnivesky-Colli C, Schweigmann NJ, Alberti A, Pietrokovsky SM, Conti O, Montoya S, et al. Sylvatic American trypanosomiasis in Argentina. Trypanosoma cruzi infection in mammals from the Chaco forest in Santiago del Estero. Trans R Soc Trop Med Hyg. 1992;86:38-41.

11. Telford SR, Tonn RJ. Dinámica de Trypanosoma cruzi en poblaciones de un reservorio primario, Didelphis marsupialis, en los llanos altos de Venezuela. Bol Oficina Sanit Panam 1982;93(4):341-364.
12. Cabrera AL. Biogeografía de América Latina. Organización de los Estados Americanos, Programa Regional de Desarrollo Científico y Tecnológico. 1980;13:69-75.

13. Cerisola JA, Rohwedder R, Segura EL, Del Prado CE, Alvarez M, Wynne de Martini GL. El xenodiagnóstico. Buenos Aires: Ministerio de Bienestar Social; 1974.

14. Zeledón R, Solano G, Saenz SG, Swartzwelder JC. Wild reservoirs of Trypanosoma cruzi with special mention of the opossum, Didelphis marsupialis, and its role in the epidemiology of Chagas'disease in an endemic area of Costa Rica. J Parasitol 1970; 56:38.

15. Barreto MP. Tripanossomos semelhantes ao Trypanosoma cruzi em animais silvestres e sua identificação com o agente etiológico da doença de Chagas. Rev Inst Med Trop Sâo Paulo 1965;7:304-315.

16. Fleiss JL. Statistical methods for rates and proportions. New York, John Wiley \& Sons, Inc.; 1981.

17. Gürtler RE, Solarz ND, Lauricella M, Haedo AS, Pietrokovsky SM, Alberti AA, et al. Dynamics of transmission of Trypanosoma cruzi in a rural area of Argentina: III, Persistence of 
Trypanosoma cruzi parasitemia among canine reservoirs in a two-year follow-up. Rev Inst Med Trop São Paulo 1986;28(4):213-219.

18. Catalá S. The biting rate of Triatoma infestans in Argentina. Med Vet Entomol 1991;5:325-333.

19. Cordero GA, Ruben A, Nicolás B. Feeding habits of the opossum (Didelphis marsupialis) in Northern Venezuela. Fieldiana: Zoology 1987;39:125-131.

20. Yaeger RG. Transmission of Trypanosoma cruzi infection to opossums via the oral route. J Parasitol 1971;57:1375-1376.

21. Ribeiro RD, Rissato e García TA, Bonoma WC. Contribução para o estudo dos mecanismos de transmissão do agente etiológico da doença de Chagas. Rev Saúde Públ. 1987; 21:51-54.

22. Wisnivesky-Colli C, Schweigmann N, Pietrokovsky S, Bottazzi V, Rabinovich JE. Spatial distribution of Triatoma guasayana (Hemiptera: Reduviidae) in hardwood forest biotopes in Santiago del Estero, Argentina. J Med Entomol.1997:34(2):102-109.

23. Romaña C. Falta de transmisión hereditaria de Trypanosoma (Schizotrypanum) cruzi en Didelphis paraguayense y comentario sobre herencia del parásito en otros mamíferos. Anales del Instituto de Medicina Regional (Tucumán, Argentina). 1955;4:149-154.

24. Zeledón R. Epidemiology, modes of transmission and reservoir hosts of Chagas disease. 20th Symposium, Ciba Foundation, Elsevier Excerpta Medica, Amsterdam, Netherlands. 1974:57-77

25. Jansen AM, Madeira FB, Deane MP. Trypanosoma cruzi infection in the opossum Didelphis marsupialis: absence of neonatal transmission and protection by maternal antibodies in experimental infections. Mem Inst Oswaldo Cruz 1994;89:41-45.

26. Deane MP, Lenzi HL, Jansen AM. Double developmental cycle of Trypanosoma cruzi in the opossum. Parasitology Today 1986;2:146-147.

27. Conti O, Schweigmann NJ, Pietrokovsky S, Bottazzi V, Wisnivesky-Colli C. Search for Trypanosoma cruzi in the anal glands of wild Didelphis albiventris from Santiago del Estero, Argentina. Mem Inst Oswaldo Cruz 1995; 90(6):687.

Manuscrito recibido el 19 de noviembre de 1998 y aceptado para publicación, tras revisión, el 30 de junio de 1999.
ABSTRACT

Study of the prevalence of infection by Trypanosoma cruzi in opossums (Didelphis albiventris) in Santiago del Estero, Argentina
The opossum of the genus Didelphis is one of the principal wild reservoirs of Trypanosoma cruzi and is widely distributed in the Western Hemisphere. Didelphis albiventris is the most common marsupial in Amamá and Trinidad, two communities in the province of Santiago del Estero, Argentina. The D. albiventris population is replaced every year, and the opossum normally has two reproductive periods, one at the beginning of the spring and another at the beginning of the summer. The two litters are weaned, and they leave the mother's marsupial pouch to join the population, the first (G1) at the beginning of the summer and the second (G2) at the beginning of the fall. Between 1988 and 1991409 D. albiventris opossums were studied, and xenodiagnoses showed that $35 \%$ of them were infected with T. cruzi. Annual cycles of renewed infection were observed, with prevalences that ranged between $22 \%$ and $43 \%$. The acquisition of the parasite occurred over the entire year, from the summer through the spring. The prevalence of infection increased with age. The G1 individuals tended to present higher prevalences than the G2 individuals, probably from being exposed to transmission for a longer period of time. In the first two (younger) age categories for the opossums, G2 individuals showed higher prevalences than did the G1 individuals. This indicates a significant increase in transmission intensity during the fall. Opossums should be regarded as a potential source of T. cruzi entry to the domestic transmission cycle. 